\title{
Estrategias didácticas para favorecer las prácticas literarias digitales en la formación inicial de maestros
}

\author{
Didactic strategies to promote digital literacy practices in initial \\ teacher training
}

\author{
Míriam Cabré Rocafort \\ Universitat de Barcelona
}

\begin{abstract}
Resumen
En la formación inicial de maestro es necesario que los estudiantes adquieran estrategias didácticas para potenciar las prácticas literarias digitales. En esta investigación se usan métodos basados en el paradigma cualitativa e interpretativa y, mediante el análisis del discurso en sus tres dimensiones (enunciativa, temática e interlocutiva), se analizan los discursos de los estudiantes acerca de una propuesta didáctica para la etapa de Primaria relacionada con el desarrollo de prácticas literarias digitales (booktrailers). Los resultados muestran que las estrategias didácticas más eficaces para el diseño de prácticas literarias digitales son: la multiliteracidad de las actividades, el aprendizaje basado en proyectos y la evaluación formadora. En conclusión, el estudio demuestra que se requiere capacitar al profesorado desde su formación inicial para diseñar propuestas didácticas que vayan más allá de la literacidad tradicional. Se trata de que adopten nuevas formas comunicativas encaminadas hacia el desarrollo de prácticas educativas que potencien la multiliteracidad.
\end{abstract}

Palabras clave: Literacidad; Aprendizaje basado en proyectos; Evaluación; Educación Primaria; Formación inicial de maestros

\begin{abstract}
In preservice teacher education it is necessary that students acquire didactic strategies to enhance digital literacy practices. In this research, methods based on the qualitative and interpretative paradigm have been used. Specifically, discourse analysis focusing on three dimensions - declarative, thematic and interlocutory - has been employed. The future teachers' discourses about a didactic activity for Primary school based on the development of digital literacy practices (booktrailers) have been interpreted. The study shows that the most efficient didactic strategies to design digital literacy practices are: multiliteracy of the activities, project based learning and formative evaluation. In conclusion, the study demonstrates that in preservice teacher education it is necessary to help students to design didactic activities that move beyond traditional literacy. They have to include new ways of communication aimed at the development of educational practices that enhance multiliteracies.
\end{abstract}

Keywords: Literacy; Project based learning; Evaluation; Primary education; Preservice teacher education 


\section{INTRODUCCIÓN}

Los futuros maestros/as de Educación Primaria tienen delante un nuevo reto: promover la lectura no sólo en entornos analógicos, sino también en los digitales. En relación al fomento de la lectura por placer en el primer tipo de contextos -los analógicos- hay mucha literatura (Bustos et al., 2017; Caride et al., 2018; Colomer y Munita, 2013; Felipe, 2016; Neira, 2015). En cambio, no hay muchos estudios sobre el fomento de la lectura en entornos digitales, aunque, especialmente desde la formación inicial de maestros/as, es necesario proporcionar estrategias didácticas al alumnado para potenciar las prácticas literarias digitales (Ballester \& Ibarra, 2016; Cassany, 2013; Warschauer, 1999).

Según estos últimos autores, las prácticas literarias digitales forman parte de la educación literaria y se caracterizan por subvertir el modelo de lectura unidireccional y de formato único, por nacer de procedimientos digitales y por desarrollarse también en medios digitales. Este cambio de modelo implica la incorporación del formato web, la incorporación de material multimodal interno y externo, y el desarrollo de tareas interactivas, entre otros aspectos novedosos. Así pues, se entiende por prácticas literarias digitales las dinámicas lectoras que conllevan una mayor interactividad (textos-lectores) e interrelación de modos de comunicación diversos (textual, visual, auditivo, etc.).

En este sentido, como apunta Cassany (2018), la literacidad está adoptando nuevas formas más allá del papel. Se está produciendo una literacidad electrónica que conlleva nuevos parámetros pragmáticos, discursivos y verbales. Por este motivo, los futuros docentes deben adquirir herramientas para que la enseñanza de la lectura incluya los discursos digitales.

Si el profesorado dispone de estas herramientas puede contribuir a la construcción de un conocimiento cultural compartido desde la Educación Primaria, de manera que el papel de la literatura sea hacer más inteligible el mundo para el alumnado (De Amo, 2003). Los futuros maestros/as tienen que ser conscientes de la importancia de educar a los estudiantes en la lectura, ya que es una de las habilidades más amplias, trascendentes y difíciles de enseñar con eficacia.

De aquí proviene la dedicación y obstinación que cualquier maestro debería poner en su enseñanza y aprendizaje en los distintos entornos. Para ello, es necesario analizar el pensamiento de los docentes acerca de la educación literaria, teniendo en cuento lo que Palou y Fons (2010) apuntan: 'reflexionar es hacer que el pensamiento vuelva sobre sí mismo para apartarse de las interpretaciones más 
inmediatas y provocar rupturas con el sentido común'. Así pues, la metacognición acerca de la educación literaria permite la atribución de sentido a las experiencias de lectura.

Esta reflexión es fundamental, porque a lo largo de la enseñanza obligatoria, durante las experiencias lectoras, se van formando conceptos como el de biblioteca interior (Bayard, 2007) o el de textoteca interna (Devetach, 2008), que apelan a la existencia de un espacio interior formado por los libros que marcan y por experiencias de lectura arraigadas profundamente y consideradas parte del imaginario personal. Estas nociones están vinculadas a las trayectorias personales de lectura y a la relación de estos bagajes con la construcción de la identidad del sujeto lector.

Para desarrollar estos y otros conceptos ligados a la educación literaria, los docentes deben reflexionar sobre su papel de mediador literario diseñando prácticas de lectura analógicas y digitales. Para conseguirlo, deben aprender a programar actividades que vayan desde la recepción individual a la que se produce en el seno de una comunidad que interpreta y valora las obras: la comunidad de lectores (Colomer, 2010). Estos espacios y puentes pueden ser analógicos o digitales, y son eficientes cuando siguen la secuencia siguiente: metacognición inicial de los conceptos clave, desarrollo de prácticas literarias y reflexión sobre las actividades para su mejora. Así, en los entornos lectores se conectan los aprendizajes con las obras, con la finalidad de aportar distintos niveles de lectura (Díaz-Plaja, 2009).

Con el propósito de desarrollar estrategias didácticas para las prácticas literarias en Educación Primaria, en el marco de la formación inicial de maestros de la Universidad de Barcelona se propuso la programación de una propuesta didáctica que potenciara la multiliteracidad, por medio de la metodología del aprendizaje basado en proyectos (ABP) y el uso de las tecnologías. Posteriormente, los propios estudiantes realizaron, a través del campus virtual, una evaluación formadora con reflexiones alrededor de los trabajos propios y de los compañeros, mediante un foro de debate.

Para comprender la metodología utilizada en este estudio es necesario poner en relieve tres conceptos clave: la multiliteracidad, el aprendizaje basado en proyectos y la evaluación formadora.

\section{La multiliteracidad}

El New London Group (Cope \& Kalantzis, 2000; New London Group, 1996) desarrolló un marco de referencia sobre las multiliteracidades, en el que expandieron la idea de ser comunicativamente competente más allá de la habilidad de leer y escribir 
en papel y en una sola lengua. Desde esta perspectiva, las prácticas lectoras deben concebirse desde una visión múltiple y situada, de acuerdo con las actuales realidades locales y globales. Tal y como demuestra Cabré (2019), en base a los postulados del New London Group (1996 y 2000), en un estudio longitudinal en el que se desplegaron métodos multimodales para investigar acerca del pensamiento de los futuros docentes, las capacidades comunicativas se desarrollan en contextos particulares, con distintos propósitos e implican el uso de modos de comunicación diversos y, a menudo, multimodales. Este estudio demuestra que la confrontación entre el conocimiento conceptual, las creencias y las prácticas educativas amplían las estrategias docentes para regular y desarrollar las prácticas educativas lectoras.

Cummins et al. (2006, p. 6) propusieron el concepto de texto identitario multimodal [multimodal identity text] para hablar de textos en los que se exprese la identidad por medio de narrativas multimodales. Este término relacionado con el de multiliteracidad permite el cuestionamiento de discursos dominantes y hegemónicos (Palou y Cabré, 2017), en el caso que nos ocupa discursos acerca de las prácticas literarias analógicas y digitales. Esta perspectiva conlleva no solo un cambio en los métodos, sino también en la manera de construir la realidad.

Además, la multiliteracidad también hace referencia al hecho de que hoy leemos distintas tipologías textuales en breves espacios de tiempo (Cassany, 2018), sobre todo en Internet, cuando pasamos rápidamente de una práctica a otra (mandar correos, leer artículos, buscar información, entre otras). Esto implica el desarrollo de nuevas competencias para cambiar rápidamente de género, lengua, temática y finalidad comunicativa.

\section{El aprendizaje basado en proyectos (ABP)}

La metodología del aprendizaje basado en proyectos (ABP), tanto en la Educación Primaria, Educación Secundaria y en la Educación Superior, plantea situaciones en las que el alumnado debe dar respuesta a un problema o reto determinado. Esta forma de aprender facilita el desarrollo de las habilidades blandas [soft skills], que son las relacionadas con la inteligencia emocional, la capacidad de comunicación, la habilidad de trabajar en equipo, la facilidad para adaptarse a los cambios, el liderazgo, el autocontrol, la productividad personal, la negociación, la resolución de conflictos y la gestión del tiempo (Peggy, 2008). Las habilidades blandas que se consideran más relevantes son las 4C: creatividad, pensamiento crítico, cooperación y comunicación. 
A pesar de que se las llame habilidades blandas, de acuerdo con Dooly \& Thorne (2018), son cada vez más importantes para aprender en el actual contexto incierto, volátil y acelerado. Estas pueden ser algunas de las competencias que, como se ha apuntado en el apartado anterior, Cassany (2018) insiste en que deben adquirirse para ser capaz de trabajar con textos multimodales. Además, el uso de estos textos en el marco del trabajo por proyectos contribuye tanto al desarrollo de las habilidades duras -el corpus de conocimiento y técnicas para adquirir saberescomo de las blandas, que se detallan a continuación.

La primera habilidad blanda, la creatividad, implica pensar y trabajar de manera creativa y colaborativa, implementando innovaciones. La segunda, el pensamiento crítico, que va ligado a la resolución de conflictos, comporta utilizar diferentes tipos de razonamiento, hacer juicios y tomar decisiones: analizar, evaluar, sintetizar, hacer conexiones y resolver problemas. La tercera y la cuarta habilidad blanda, la cooperación y la comunicación, comportan saber comunicar, de manera clara, lo que se quiere expresar a través de varios medios (orales, escritos, visuales, auditivos, etc.), en diferentes contextos y con distintas finalidades. Así mismo, cooperar permite desarrollar flexibilidad, voluntad, respeto y ética (Giusti, 2008).

\section{La evaluación formadora}

La finalidad de la evaluación es regular los procesos de enseñanza y aprendizaje para detectar dificultades y errores, y así tomar medidas para progresar (Sanmartí, 2007). Esta evaluación se llama formativa cuando la realiza el profesorado y formadora cuando la lleva a cabo el alumnado. Para potenciar la evaluación formadora es imprescindible la representación de los objetivos de aprendizaje y la valoración de los aprendizajes (Villalonga \& Deulofeu, 2015).

En esta investigación, mediante el foro del campus virtual los estudiantes llevan a cabo valoraciones acerca de la propuesta de actividad del booktrailer, un vídeo breve para promocionar o anticipar una obra, teniendo en cuenta elementos como el libro, el título, el texto, la música, los efectos de sonido, el ritmo de las secuencias, el tono, el contexto, los elementos visuales, la voz narrativa y los elementos narrativos. Todos ellos para reflexionar sobre el paso de la literacidad tradicional a la multiliteracidad mediante prácticas literarias digitales (véase en la tabla 5 los bloques temáticos emergidos en las intervenciones de los estudiantes).

En conclusión, si observamos los tres conceptos clave desde la perspectiva sociocultural (Vygotsky, 1978), las tres estrategias didácticas -la multiliteractidad, el aprendizaje por proyectos y la evaluación formadora- provocan cambios en las 
prácticas literarias digitales y, en general, en la educación literaria del alumnado. Esto es así porque estas estrategias implican un tipo de aprendizaje que conlleva la interacción entre los individuos y los entornos -sean analógicos o digitales-. Estos procesos interactivos favorecen la reconstrucción, tanto de los conocimientos como de las prácticas y las experiencias de lectura y escritura. Así pues, la interacción se convierte en un instrumento de primer orden, puesto que todo proceso de aprendizaje es fruto de un proceso de mediación.

\section{MÉTODO}

\section{Objetivos de investigación}

Los objetivos del estudio son:

1. Analizar estrategias didácticas que promuevan prácticas de lectura en entornos digitales.

2. Identificar qué tipo de narrativas surgen de las propuestas didácticas de lectura digital.

3. Señalar algunas de las implicaciones de estas narrativas en la formación inicial de maestros y la educación literaria en los niveles iniciales.

\section{Paradigma de investigación, instrumentos y participantes}

El paradigma de investigación en el que se enmarca esta investigación es el cualitativo-interpretativo. Se utiliza el análisis del discurso para acercarse al diseño de prácticas literarias digitales en la formación inicial de maestros. En este estudio, la interpretación del discurso permite acceder a las narrativas de los estudiantes derivadas de la interacción entre estos y las actividades de lectura digitales. Esta metodología permite entender los discursos de los participantes a partir de su propia subjetividad y el contexto en el que se da la interacción entre estos y sus formas de expresión.

El enfoque de esta investigación es el narrativo en educación, que se desarrolla a través de un proceso de recogida de datos discursivos. El corpus de datos está formado por las intervenciones en un foro de debate electrónico de 39 futuros maestros/as, que emergen de la reflexión sobre las propias tareas académicas acerca de la educación literaria en entornos digitales. Las características principales de los participantes son: estudiantes del tercer curso del Grado en Educación Primaria de la Universidad de Barcelona, mayoritariamente de sexo femenino, y de entre 20 y 26 años. 
El principal instrumento de investigación son las intervenciones de los maestros/as en formación inicial, que se repartieron en nueve grupos de trabajo. Cada equipo debía hacer un mínimo de una intervención en el foro, con la finalidad de llevar a cabo una evaluación formadora. Esta evaluación implicó reflexionar acerca de una tarea académica centrada en el diseño de una propuesta didáctica para fomentar las prácticas literarias digitales (booktrailer). Para este estudio se ha seleccionado una intervención de cada grupo.

En definitiva, este tipo de investigación ofrece posibilidades de investigar los procesos de expresión e interpretación, así como el desarrollo de las competencias profesionales relacionadas con la adquisición de las competencias lectoras y literarias en Educación Primaria.

\section{Datos de la investigación}

En la tabla 1 se muestran los datos recogidos para este estudio.

Tabla 1. Intervenciones de los estudiantes del Grado de Educación Primaria en el campus virtual

1. Trabajar la lectura con las tecnologías nos parece acertado, porque conlleva que los alumnos tengan que usar dispositivos para hacer fotografías o grabar vídeos, editarlos, descargarse música y sonido, etc., para elaborar su booktrailer. Así pensamos que adquieren destreza y comodidad con las TIC.

2. Los booktrailers ayudan a aprender a comprender e interpretar textos literarios. Para su realización es imprescindible que todos los alumnos entiendan el texto que tienen que transformar a otro lenguaje. Para conseguirlo deben comunicarse y negociar posibles interpretaciones, malentendidos y simbolismos adheridos.

3. Otra ventaja de plantear el trabajo por proyectos a través de la realización de booktrailers es que enseña a extraer las ideas principales de las obras literarias. La realización del vídeo implica que los estudiantes seleccionen qué quieren contar: decidir qué es importante y esencial del texto. Eso lleva a que todos los miembros del grupo tengan que ponerse de acuerdo sobre lo que quieren explicar y lo que descartan, de manera que se desarrollan conversaciones donde cada uno tendrá que justificar su elección. De este modo, los niños y niñas aprenden a formular justificaciones razonadas con argumentos sólidos.

4. Otro aspecto positivo del uso del ABP para la elaboración de los booktrailers es que los alumnos adquieren destrezas para reinterpretar los textos literarios en otros lenguajes. Los estudiantes tienen que pasar del formato textual al del vídeo, un traspaso de lenguajes e informaciones que comporta cierto control del relato. A través de la metodología del trabajo por proyectos, se consigue que los alumnos investiguen y encuentren la mejor manera de expresar aquello que quieren comunicar acerca de la lectura para convencer a sus iguales.

5. El trabajo del proyecto del booktrailer permite introducir desde Primaria los conceptos de multiliteracidad y gusto por la lectura, que consideramos esenciales para la educación del ámbito lingüístico y literario. 


\begin{abstract}
6. Con la elaboración del booktrailer nos alejamos de la idea tradicional de solo trabajar la lengua a través de la escritura y la lectura. En este sentido, pensamos que la elaboración de un proyecto de este tipo es una buena estrategia para que los alumnos utilicen lenguajes y medios de expresión diversos, como la música, el dibujo, la fotografía, etc. De este modo, se trabaja la multiliteracidad a través del desarrollo de los proyectos, y se respetan las distintas formas de aprender de los niños, es decir, descubren con cuál se sienten más cómodos. Por último, pensamos que a través de este tipo de metodología, podrán descubrir los límites y posibilidades de los lenguajes explorando el mejor modo de expresar aquello que quieren contar.
\end{abstract}

7. Nuestro grupo se ha fijado en que los booktrailers permiten fomentar el gusto por la lectura, ya que es una forma original e impactante de presentar un libro. Creemos que es así porque explica la trama sin desvelar el final de la historia, dejando así oportunidades a los demás para imaginar posibles desenlaces.

8. El proyecto acerca de la elaboración de booktrailers permite que los alumnos trabajen en equipo para hacer una tarea que atraiga, interese y cautive a futuros lectores. También relacionado con el gusto por la lectura, pensamos que la selección del libro no debe ser casual, ya que debe permitir la reelaboración del texto literario a otro lenguaje.

9. En la metodología del trabajo por proyectos usando las tecnologías para hacer un booktrailer tiene mucha importancia el trabajo en equipo y la cohesión de sus miembros. Este tipo de método didáctico comporta momentos de trabajo individual, pero realmente estos son pocos. La mayor parte del tiempo se trabaja en grupo, para ponerse de acuerdo, distribuir tareas y desarrollarlas conjuntamente.

\title{
Procedimiento
}

Para la recogida de datos se han utilizado consignas de tipo no direccional (Bertaux, 2005), ya que favorecen la reelaboración libre de la experiencia y permiten obtener un panorama general del tema. Estas pueden resumirse en los siguientes puntos:

En el marco de la asignatura Didáctica de la Literatura Infantil y Juvenil del Grado de Educación Primaria de la Universidad de Barcelona, se pide al alumnado que elabore una propuesta didáctica para desarrollar el gusto por la lectura a través del uso de las tecnologías, con los siguientes apartados: título, objetivos generales y específicos, desarrollo, recursos y temporalización, criterios de evaluación y bibliografía. Este trabajo se plantea para ser realizado en grupos de tres o cuatro estudiantes y mediante la metodología del aprendizaje por proyectos (ABP). Esta metodología implica diseñar una propuesta para fomentar las prácticas literarias digitales (reto), que conlleve un proceso de búsqueda, tratamiento y comunicación de la información y del resultado final (booktrailer).

Posteriormente, se anima a los estudiantes a que reflexionen a través del foro de debate del campus virtual acerca de sus propuestas y las de los compañeros después de las presentaciones orales de las propuestas didácticas-. En concreto, la 
docente invita a reflexionar sobre el potencial de las propuestas didácticas para el desarrollo de prácticas literarias digitales en Primaria, haciendo hincapié en los conceptos de multiliteracidad, aprendizaje basado en proyectos y uso de las tecnologías. De este modo, se lleva a cabo una evaluación formadora entre iguales y formativa por parte de la docente.

\section{Análisis de datos}

Los criterios para el análisis de datos discursivos se basan en los resultados de las investigaciones en el ámbito de la didáctica de la lengua y la literatura, en concreto las aportaciones de Kerbrat-Orecchioni (2005), Pavlenko (2007), Riessman (2008), Palou y Fons (2010) y Melo-Pfeifer \& Schmidt (2012). En este sentido, el análisis se focaliza en tres dimensiones de los textos, que están interrelacionadas: interlocutiva, temática y enunciativa (véase Tabla 2).

Tabla 2. Categorías para el análisis del discurso (Cabré, 2018)

\begin{tabular}{ll}
\hline $\begin{array}{c}\text { Dimensiones del análisis } \\
\text { del discurso }\end{array}$ & \multicolumn{1}{c}{ Elementos de análisis } \\
\hline $\begin{array}{l}\text { Dimensión interlocutiva } \\
\text { (Cómo) }\end{array}$ & $\begin{array}{l}\text { Marco de participación, organización del discurso, aspectos con- } \\
\text { textuales y polifonía (distintas voces, es decir, puntos de vista que } \\
\text { aparecen en el relato). }\end{array}$ \\
$\begin{array}{l}\text { Dimensión temática } \\
\text { (Qué) }\end{array}$ & $\begin{array}{l}\text { Análisis de los temas y las palabras clave. Valoración de los sujetos } \\
\text { acerca de las prácticas literarias digitales. }\end{array}$ \\
$\begin{array}{l}\text { Dimensión enunciativa } \\
\text { (Posición) }\end{array}$ & $\begin{array}{l}\text { Posicionamiento enunciativo (posición del sujeto respecto a lo que } \\
\text { dice), interlocutores y situación comunicativa, puntos críticos (indi- } \\
\text { cios de cambio que marcan un antes y un después en el aprendi- } \\
\text { zaje) y recursos expresivos o metafóricos. }\end{array}$ \\
\hline
\end{tabular}

\section{RESUlTADOS}

Los resultados se estructuran según los elementos de las tres dimensiones -interlocutiva, temática y enunciativa- (véase Tabla 2) del análisis de discurso, propias del ámbito de la didáctica de la lengua y la literatura. Algunos de los elementos analizados se ejemplifican con los datos de la investigación.

\section{Dimensión interlocutiva}

Marco de participación. Al tratarse de una tarea de una asignatura del Grado de Educación Primaria, los discursos se dirigen a los compañeros de la asignatura y a la docente. 
Organización del discurso. Los discursos del alumnado son breves, ya que se trata de intervenciones valorativas acerca del trabajo por proyectos para elaborar booktrailers. Algunas reflexiones tienen resonancias, de modo que tratan los mismos bloques temáticos. Así mismo, cabe destacar que hay intervenciones que interrelacionan diferentes temas, como por ejemplo el gusto por la lectura y el trabajo por proyectos (véase Tabla 3).

Tabla 3. Ejemplo de la interrelación de temas (trabajo en equipo, gusto por la lectura y transformación de lenguajes)

\begin{abstract}
8. El proyecto acerca de la elaboración de booktrailers permite que los alumnos trabajen en equipo para hacer una tarea que atraiga, interese y cautive a futuros lectores. También relacionado con el gusto por la lectura, pensamos que la selección del libro no debe ser casual, ya que debe permitir la reelaboración del texto literario a otro lenguaje.
\end{abstract}

Aspectos contextuales. El ámbito vital de los discursos de los futuros docentes es el académico, ya que las intervenciones hacen referencia a las comunidades de práctica escolar y profesional.

Polifonía. En los discursos de los estudiantes aparecen sus voces como futuros maestros/as (véase Tabla 4), pero estas conviven con otras, como la de los niños/as de Educación Primaria, que son los destinatarios de las propuestas de los booktrailers.

Tabla 4. Ejemplo de polifonía

3. Otra ventaja de plantear el trabajo por proyectos a través de la realización de booktrailers es que enseña a extraer las ideas principales de las obras literarias. La realización del vídeo implica que los estudiantes seleccionen qué quieren contar: decidir qué es importante y esencial del texto. Eso lleva a que todos los miembros del grupo tengan que ponerse de acuerdo sobre lo que quieren explicar y lo que descartan, de manera que se desarrollan conversaciones donde cada uno tendrá que justificar su elección. De este modo, los niños y niñas aprenden a formular justificaciones razonadas con argumentos sólidos.

\title{
Dimensión temática
}

Temáticas y palabras clave. Los conceptos clave permiten observar hacia dónde se orientan los discursos de los futuros docentes y, así, descubrir los temas principales y los mecanismos sociales que influyen en la percepción de los estudiantes acerca de las prácticas literarias digitales. En este caso, los siete bloques temáticos de la Tabla 5, que han emergido de las intervenciones del foro, son los más relevantes 
para los estudiantes. Estos se estructuran alrededor de las palabras clave señaladas en la tabla 5 .

Tabla 5. Bloques temáticos de los discursos

1. Uso de las tecnologías.

2. Transformación de lenguajes.

3. Multiliteracidad.

4. Trabajo basado en proyectos (ABP).

5. Gusto por la lectura.

6. Trabajo en equipo.

7. Desarrollo de la competencia comunicativa (que incluye la lectora).

Después de un análisis detallado de las categorías, se ha observado que la más significativa es la segunda -la transformación de lenguajes-, seguida de la cuarta -el trabajo por proyectos-. En menor medida, aparecen la tercera, la quinta y la séptima categoría -la multiliteracidad, el gusto por la lectura y el desarrollo de la competencia comunicativa, respectivamente-. Y, por último, las categorías con menor relevancia para los futuros maestros/as son la primera y la sexta, es decir, el uso de las tecnologías y el trabajo en equipo. En la Tabla 6 se muestra el análisis cuantitativo de las categorías temáticas.

Tabla 6. Análisis cuantitativo de los bloques temáticos

\begin{tabular}{ll}
\hline \multicolumn{1}{c}{ Bloques temáticos } & $\begin{array}{c}\mathrm{N}^{\circ} \text { de intervenciones del foro } \\
\text { (véase anexos) }\end{array}$ \\
\hline 1. Uso de las tecnologías. & 2 intervenciones (1 y 9). \\
2. Transformación de lenguajes. & 5 intervenciones $(1,2,4,6$ y 8). \\
3. Multiliteracidad. & 3 intervenciones $(1,5$ y 6$)$. \\
4. Trabajo basado en proyectos (ABP). & 4 intervenciones (3, 4, 6 y 9). \\
5. Gusto por la lectura. & 3 intervenciones (5, 7 y 8). \\
6. Trabajo en equipo. & 2 intervenciones (8 y 9). \\
7. Desarrollo de la competencia comunicativa. & 3 intervenciones (2, 3 y 4). \\
\hline
\end{tabular}

Valoración acerca de las prácticas literarias digitales. En los discursos del alumnado de Grado se percibe una valoración positiva acerca de cómo la metodología del trabajo por proyectos para elaborar el booktrailer favorece la adquisición de capacidades para el uso de las tecnologías, la transformación entre formas de comunicación y lenguajes, el trabajo en equipo, el fomento del gusto por la lectura y el desarrollo de la competencia comunicativa en todas sus dimensiones. 


\section{Dimensión enunciativa}

Posicionamiento enunciativo. En el relato se identifican valoraciones respecto a los beneficios de la metodología basada en los proyectos para elaborar booktrailers. Estos son: el desarrollo de la competencia comunicativa -también lectora y literaria- y el gusto por la lectura. Así mismo, se identifican apreciaciones acerca de las contribuciones de estas prácticas literarias digitales para fomentar la cohesión de grupo, la transformación entre lenguajes y formas de comunicación y para la adquisición de destrezas relacionadas con la competencia digital (véase Tabla 7).

Tabla 7. Ejemplos del posicionamiento enunciativo

1. Trabajar la lectura con las tecnologías nos parece acertado, porque conlleva que los alumnos tengan que usar dispositivos para hacer fotografías o grabar vídeos, editarlos, descargarse música y sonido, etc., para elaborar su booktrailer. Así pensamos que adquieren destreza y comodidad con las TIC.

2. Los booktrailers ayudan a aprender a comprender e interpretar textos literarios. Para su realización es imprescindible que todos los alumnos entiendan el texto que tienen que transformar a otro lenguaje. Para conseguirlo deben comunicarse y negociar posibles interpretaciones, malentendidos y simbolismos adheridos.

Todo ello se expresa desde tres posicionamientos: el personal, el de maestro/a (futura profesión), y el de alumno/a de Educación Primaria. La modalidad oracional más frecuente es la enunciativa-afirmativa y las conductas discursivas que aparecen son la exposición y la argumentación, las cuales les permiten informar, situar consideraciones teóricas y hacer comprensibles los puntos críticos de sus discursos acerca de las prácticas lectoras digitales. Por último, las modalidades verbales predominantes son el presente y el pasado, lo cual es coherente con los tipos de discursos, explicativos y argumentativos, sobre las propuestas didácticas.

Interlocutores y situación comunicativa. En los discursos predomina la tercera persona del plural, porque las intervenciones corresponden a los grupos de trabajo de las propuestas didácticas. Estos equipos de trabajo interaccionan entre ellos a través del foro del campus virtual de la Universidad.

Puntos críticos. En el texto hay cuatro puntos críticos (véase Tabla 8) que relatan sucesos que marcan un antes y un después en la valoración de la propuesta didáctica acerca del trabajo por proyectos para la elaboración del booktrailer. 
Tabla 8. Puntos críticos del discurso e intervenciones donde aparecen

\begin{tabular}{ll}
\hline \multicolumn{1}{c}{ Puntos críticos } & \multicolumn{1}{c}{$\begin{array}{c}\text { Intervenciones del foro en las que } \\
\text { se desarrollan (véase Tabla 1) }\end{array}$} \\
\hline $\begin{array}{l}\text { 1. Trabajar la lectura con las tec- } \\
\text { nologías. }\end{array}$ & Intervenciones 1 y 9. \\
2. La importancia de tener com- & Intervenciones 2, 3, 4, 6 y 8. \\
petencia comunicativa para & \\
transformar lenguajes y formas & \\
de comunicación. & \\
3. El uso del trabajo basado en & Intervenciones 3, 4, 5, 6, 7 y 8. \\
proyectos (ABP) para elaborar & \\
booktrailers contribuye al paso \\
de la idea tradicional de literaci- \\
dad al de multiliteracidad, y po- \\
tencia el fomento del gusto por \\
la lectura. \\
$\begin{array}{l}\text { 4. La metodología por proyectos } \\
\text { para realizar booktrailers tiene }\end{array}$ \\
$\begin{array}{l}\text { como requisito la cohesión y el } \\
\text { trabajo en equipo. }\end{array}$ \\
\hline
\end{tabular}

Recursos expresivos y metafóricos. Al analizar las valoraciones de los futuros docentes se aprecia el uso de términos calificativos, como por ejemplo: acertado (el uso de la tecnología), conceptos esenciales (la multiliteracidad y el gusto por la lectura), buenas estrategias (el ABP y los booktrailers), sentirse cómodos/el mejor modo (para hablar de la transformación entre lenguajes), forma original e impactante (de presentar un libro), destreza y comodidad (relacionadas con la competencia digital), justificaciones razonadas y argumentos sólidos (para el trabajo en equipo), y tarea que atraiga, interese y cautive a futuros lectores (la del booktrailer).

En relación con los recursos metafóricos, solo hay una metáfora incipiente que es la de idea tradicional de aprender lengua solo a través de la lectura y escritura en papel, la cual ayuda a los futuros maestros/as a contraponerla a la de prácticas literarias digitales.

\section{DISCUSIÓN Y CONCLUSIONES}

Como se ha destacado a través de los resultados, el desarrollo de esta dinámica basada en el aprendizaje por proyectos para la realización del booktrailer y la posterior evaluación formadora -con la participación en un foro de debate- ha proporcionado estrategias didácticas eficaces para desarrollar prácticas literarias digitales en Educación Primaria. De este modo, se ha cumplido el primer objetivo y la 
pregunta de investigación acerca de las estrategias didácticas que favorecen las prácticas literarias digitales, las cuales se discuten a continuación.

\section{La multiliteracidad}

Como se desprende de los resultados, la multiliteracidad implica el uso de distintos lenguajes (verbales, visuales, digitales, auditivos y performativos), para poner en práctica diferentes formas de expresión literaria y atribuir diferentes significados a las experiencias lectoras. Esto significa que desarrollar la competencia literaria va más allá de la habilidad de leer y escribir en papel, porque implica adoptar una visión múltiple y situada de las prácticas alfabetizadoras de acuerdo con las realidades globales e interconectadas, tal y como autores precedentes habían apuntado (Cope \& Kalantzis, 2000).

Por lo tanto, desde este punto de vista, la multiliteracidad implica que la alfabetización desde las primeras etapas de la formación obligatoria debe entenderse como un repertorio de prácticas cambiantes usadas con fines comunicativos en contextos múltiples. Esto significa que no es una competencia o un conjunto de habilidades adquiridas por el alumnado y aplicables universalmente, sino que se trata de un conjunto de prácticas dinámicas -en este caso, sobre todo literarias digitales- que tienen lugar en contextos particulares, con distintos propósitos y que conllevan el uso de modos de comunicación diversos.

Así pues, dando respuesta al segundo y tercer objetivo y preguntas de investigación, es necesario preparar al profesorado desde la formación inicial para ser capaz de diseñar prácticas literarias en Educación Primaria, tanto digitales como tradicionales. Estas deben centrar la atención en la intencionalidad y la creación de producciones basadas en la multiliteracidad -como los booktrailers-, en las que los significados sean construidos con diferentes patrones modales (textual, visual, auditivo, performativo, etc.). En este sentido, se produce lo que Cassany (2018) llama literacidad electrónica, juntamente con la tradicional y, en consecuencia, se incluyen en las prácticas educativas los discursos digitales para el fomento de la lectura.

\section{El aprendizaje basado en proyectos (ABP)}

A partir de esta investigación, los datos sugieren que la metodología basada en proyectos contribuye al aprendizaje de las siguientes competencias: saber analizar una situación para ofrecer una propuesta de resolución; buscar información, analizarla y adaptarla para un propósito; organizar y gestionar tareas, y compartir con otros los trabajos realizados. En el caso del booktrailer, los estudiantes han 
analizado cómo fomentar la lectura digital en Educación Primaria (propuesta de resolución); han hecho un proceso de búsqueda, tratamiento y comunicación de la información sobre una lectura concreta, y han pensado actividades destinadas a los estudiantes de las primeras etapas, que han sido compartidas (booktrailers disponibles en el campus virtual de la asignatura).

Algunas de las implicaciones del uso del ABP en la formación de maestros/as y en Educación Primaria es que los proyectos permiten desarrollar de forma contextualizada las habilidades ligadas a la competencia comunicativa -que comprende la lectora y literaria- y fomentar habilidades transversales para utilizar la lectura y la escritura para satisfacer las propias necesidades e intereses. En el caso concreto de esta investigación, los futuros maestros/as han usado el ABP para desarrollar una propuesta didáctica para fomentar prácticas literarias digitales, específicamente un booktrailer dirigido a la etapa de Educación Primaria.

\section{La evaluación formadora}

El uso del foro de debate del campus virtual para la evaluación formadora ha mostrado ser efectivo, teniendo en cuenta que ha permitido recoger datos, analizarlos e interpretarlos, así como tomar decisiones. Estas tres acciones que, según Sanmartí (2007), ha de aportar toda evaluación, ha permitido que los estudiantes participaran del proceso de evaluación, de modo que no solo fuera formativo y calificativoacreditativo, es decir, realizado por la docente. A través de la evaluación formadora entre iguales, los futuros maestros/as han llevado a cabo procesos metacognitivos acerca de las prácticas literarias digitales propuestas durante su formación inicial (Palou \& Fons, 2010).

Además, las tres estrategias didácticas remarcadas contribuyen a capacitar a los futuros docentes para diseñar propuestas didácticas que permitan la formación de la biblioteca interior (Bayard, 2007) o textoteca interna (Devetach, 2008) del alumnado. De este modo, se ha incidido tanto en la importancia de contribuir a la formación de la identidad lectora de los estudiantes como en el papel de mediador literario (Colomer, 2010) del profesorado.

En definitiva, los resultados expuestos, a pesar de basarse en una experiencia limitada y muy contextualizada, demuestran que la propuesta de los booktrailers a través del aprendizaje basado en los proyectos es acertada, ya que da la oportunidad de elaborar experiencias compartidas con sentido para desplegar prácticas literarias digitales. Investigaciones como esta abren nuevas líneas de futuro hacia una pedagogía de las multiliteracidades literarias vinculadas a la era digital. Así 
pues, tal como se afirma desde el paradigma socioconstructivista (Vygotsky, 1978), tiene que haber un verdadero motivo en la lectura y la escritura, es decir, sentido en el acto comunicativo durante el desarrollo de prácticas literarias.

\section{REFERENCIAS}

Ballester, J. \& Ibarra, N. (2016). La educación lectora, literaria y el libro en la era digital. Revista chilena de literatura, 94. https://doi.org/10.4067/S0718-22952016000300008

Bayard, P. (2007). Comment parler des libres que l'on n'a pas lus. Minuit.

Bertaux, D. (2005). Los relatos de vida. Perspectiva etnosociológica. Edicions Bellaterra.

Bustos, A., Montenegro, C., Tapia, A., \& Calfual, K. (2017). Leer para aprender: Cómo interactúan los profesores con sus alumnos en la Educación Primaria. Ocnos, 16(1), 89-106. https://doi.org/10.18239/ocnos_2017.16.1.1208

Cabré, M. (2018). La competència plurilingüe en la formació inicial de mestres. Estudi longitudinal de casos sobre l'evolució de les creences relacionades amb l'educació plurilingüe. [Tesi Doctoral]. Universitat de Barcelona. http://hdl.handle.net/10803/666996

Cabré, M. (2019). The development of the plurilingual education through multimodal reflection in Teacher Education: A case study of a pre-service teacher's beliefs about language education. Canadian Modern Language Review, 75(1), 40-64. http://doi.org/10.3138/cmlr.2017-0080

Caride, J. A., Carballo, M. B., \& Gradaílle, R. (2018). Leer en tiempos de ocio: los estudiantes, futuros profesionales de la educación, como sujetos lectores. Ocnos, 17(3), 7-18. https://doi.org/10.18239/ocnos_2018.17.3.1707

Cassany, D. (2013). ¿Cómo se lee y escribe en línea? Revista Electrónica: Leer, Escribir y Descubrir [RELED]. International Reading Association, 1(1), 1-24.

Cassany, D. (2018). Investigaciones y propuestas sobre literacidad actual: multiliteracidad, Internet y criticidad. En Cátedra UNESCO para la Lectura y la Escritura (pp. 1-10). Universidad de Concepción.

Colomer, T. (2010). Introducción a la literatura infantil y juvenil. Síntesis.

Colomer, T., \& Munita, F. (2013). La experiencia lectora de los alumnos de Magisterio: nuevos desafíos para la formación docente. Lenguaje y Textos, 38, 37-44.

Cope, B., \& Kalantzis, M. (2000). Multiliteracies: Literacy learning and the Design of Social Futures. Taylor and Francis. http://doi.org/10.2307/358703

Cummins, J., Bismilla, V., Chow, P., Cohen, S., Giampapa, F., Leoni, L., Leoni, L., Sandhu, P., \& Padma, S. (2006). ELL students speak themselves: Identity texts and literacy engagement in multilingual classrooms (Manuscrito no publicado).

De Amo, J. M. (2003). Literatura Infantil: claves para la formación de la competencia Literaria. Ediciones Aljibe.

Devetach, L. (2008). La construcción del camino lector. Comunicarte.

Díaz-Plaja, A. (2009). Los estudios de literatura infantil y juvenil en los últimos años. Textos de Didáctica de la Lengua y la Literatura, 51, 17-28.

Dooly, M. \& Thorne, S. L. (2018). Knowledge for network-based education, cognition \& teaching: Key competences for the 21st century. KONECT White paper. https://doi.org/10.6084/m9.figshare.7366982

Felipe, A. (2016). Competencia, estrategias y hábitos lectores de maestros en formación inicial [Tesis doctoral]. Universidad de Málaga. 
Giusti, G. (2008). Soft Skills for Lawyers. Chelsea Publishing.

Kerbrat-Orecchioni, C. (2005). Le discours en interaction. Armand Universitaires de France. https://doi.org/10.1017/S0047404508080792

Melo-Pfeifer, S., \& Schmidt, A. (2012). Linking 'Heritage Language’ Education and Plurilingual repertories development: evidences from drawings of Portuguese pupils in Germany. L1Educational Studies in Language and Literature, 12, 1-30.

Neira, M. R. (2015). La lectura como actividad de ocio en escolares asturianos de $6^{\circ}$ de Primaria. Didáctica. Lengua y Literatura, 27, 131-151. https://doi.org/10.5209/rev_DIDA.2015.v27.50869

New London Group. (1996). A pedagogy of mulitliteracies: Designing social futures. Harvard Educational Review, 66(1), 60-94. http://dx.doi.org/10.17763/haer.66.1.17370n67v22j160u

Palou, J., \& Cabré, M. (2017). Beliefs regarding plurilingual competence: the perspective in Catalonia through a case study of a pre-service teacher. Modern Journal of Language Teaching Methods (MJLTM), 7(8), 63-75.

Palou, J., \& Fons, M. (2010). Metacognició i relats de vida lingüística en els processos de formació del professorat. Anàlisi del relat d'una alumna. Dins Institut de Ciències de l'Educació Josep Pallach (Ed.), Multilingüisme i pràctica educativa (pp. 255-260). Universitat de Girona.

Pavlenko, A. (2007). Autobiographic Narratives as Data in Applied Linguistics. Applied Linguistics, 28(2), 163-188. http://doi.org/10.1093/applin/amm008

Peggy, K. (2008). The Hard Truth About Soft Skills: Workplace Lessons Smart People Wish They'd Learned Sooner. Harper Collins.

Riessman, C. K. (2008). Narrative Methods for the Human Sciences. Sage.

Sanmartí, N. (2007). Resum del llibre 10 ideas clave. Evaluar para aprender. Graó.

Villalonga, J., \& Deulofeu, J. (2017). La base de orientación en la resolución de problemas: "Cuando me bloqueo o me equivoco". REDIMAT, 6(3), 256-282. http://doi.org/10.1783/redimat.2017.2262

Vygotsky, L. S. (1978). Mind in society. The development of higher psychological processes. Harvard University Press.

Warschauer, M. (1999). Electronic Literacies: Language, Culture, and Power in Online Education. Erlbaum.

\section{MÍRIAM CABRÉ ROCAFORT}

Doctora en Didáctica de las Lenguas (2018) y profesora del Departamento de Educación Lingüística y Literaria, de la Universidad de Barcelona. Miembro del grupo Plurilingüismos Escolares y Aprendizaje de Lenguas (PLURAL). Forma parte del grupo de investigación consolidado LLETRA. Combina la docencia y la investigación en la Universidad con la tarea de maestra de Primaria.

miriamcabre8@gmail.com https://orcid.org/0000-0002-2749-0121 
Cabré Rocafort, M. (2021). Estrategias didácticas para favorecer las prácticas literarias digitales en la formación inicial de maestros. Bellaterra Journal of Teaching \& Learning Language \& Literature, 14(3), e891. https://doi.org/10.5565/rev/jtl3.891

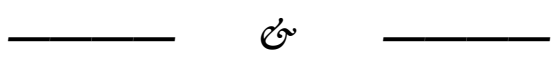

Rebut / Recibido / Received / Reçu: 13-01-2020

Acceptat / Aceptado / Accepted / Accepté: 10-10-2021

https://revistes.uab.cat/jtl3/ 